\title{
Impedance model for the polarization-dependent optical absorption of superconducting single-photon detectors
}

\author{
E.F.C. Driessen ${ }^{1}$, F.R. Braakman ${ }^{1}$, E.M. Reiger ${ }^{2}$, S.N. Dorenbos ${ }^{2}$, V. Zwiller ${ }^{2}$, and M.J.A. de Dood ${ }^{1, a}$ \\ 1 Huygens Laboratory, Leiden University, P.O. Box 9504, 2300 RA Leiden, The Netherlands \\ ${ }^{2}$ Kavli Institute for Nanoscience, Delft University of Technology, Lorentzweg 1, 2628 CE Delft, The Netherlands
}

Received: 20 February 2009 / Accepted: 9 March 2009

Published online: 14 May 2009 - C EDP Sciences

\begin{abstract}
We measured the single-photon detection efficiency of NbN superconducting single-photon detectors as a function of the polarization state of the incident light for different wavelengths in the range from $488 \mathrm{~nm}$ to $1550 \mathrm{~nm}$. The polarization contrast varies from $\sim 5 \%$ at $488 \mathrm{~nm}$ to $\sim 30 \%$ at $1550 \mathrm{~nm}$, in good agreement with numerical calculations. We use an optical-impedance model to describe the absorption for polarization parallel to the wires of the detector. For the extremely lossy NbN material, the absorption can be kept constant by keeping the product of layer thickness and filling factor constant. As a consequence, the maximum possible absorption is independent of filling factor. By illuminating the detector through the substrate, an absorption efficiency of $\sim 70 \%$ can be reached for a detector on Si or GaAs, without the need for an optical cavity.
\end{abstract}

PACS. 85.25.-j Superconducting devices - 13.88.+e Polarization in interactions and scattering

\section{Introduction}

Superconducting single-photon detectors (SSPDs) [1], that consist of a meandering $\mathrm{NbN}$ wire, are an interesting new class of detectors that may outperform singlephoton counting avalanche photodiodes. SSPDs feature a relatively high quantum efficiency at infrared wavelengths, combined with low time jitter, low dark counts, and high counting rates [2]. This makes these detectors promising for quantum optical studies and long-distance quantum cryptography applications [3].

A lot of attention has been given to the electronic operation of these detectors [4-6], and the exact microscopic working principle of the detectors is still under active investigation $[7,8]$. On a macroscopic level, a photon that is absorbed by the superconducting wire triggers a temporary loss of superconductivity, which gives rise to a finite voltage pulse across the detector. Thus, the optical absorption efficiency plays a key role and is primarily determined by the geometry of the detector and the dielectric constant of the NbN layer. Since the energy of the incident photons is much larger than the superconducting gap of the $\mathrm{NbN}$, the complex dielectric constant of the $\mathrm{NbN}$ layer at room temperature can be used [9].

Due to the highly anisotropic nature of the wires, the detection efficiency shows a strong polarization dependence $[10,11]$. This is either undesirable in experiments

\footnotetext{
a e-mail: mdedood@molphys.leidenuniv.nl
}

with unpolarized light, or the effect can be exploited to optimize the detection efficiency for polarized photons. This polarization dependence of NbN SSPDs has been reported at a single wavelength of $1550 \mathrm{~nm}$ [10] for different detector geometries. The polarization dependence of the detectors was found to be largely independent of wavelength for strongly focused light in a narrow range of wavelengths around $800 \mathrm{~nm}$ [11]. Here we report (see Sect. 3) a strong wavelength dependence of the polarization contrast, for a wavelength range from 488 to $1550 \mathrm{~nm}$. This wavelength dependence is different from the cavity enhancement reported for a $\mathrm{NbN}$ detector inside a cavity [10,12], where the wavelength dependence originates from the resonant effect of the cavity.

We introduce an analytical optical impedance model in Section 4 to gain physical insight in the optical absorption of thin lossy films. We describe different ways to increase the detection efficiency by changing the parameters of the detector. We find that the optimum thickness depends on the polarization and is a strong function of the filling factor. However, the maximum achievable absorption is independent of the $\mathrm{NbN}$ filling factor. The optical impedance model also provides more insight into the cavity enhancement reported for a $\mathrm{NbN}$ detector inside a Fabry-Perot type cavity $[10,12]$. We show, in Section 4.2 , that the absorption of the detector is enhanced by a factor $n$, with $n$ the refractive index of the substrate, when the detector is illuminated from the substrate. This factor was not accounted for in earlier work and leads to an overestimate 


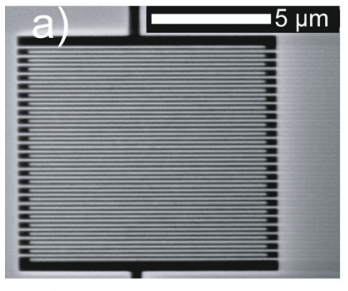

c)

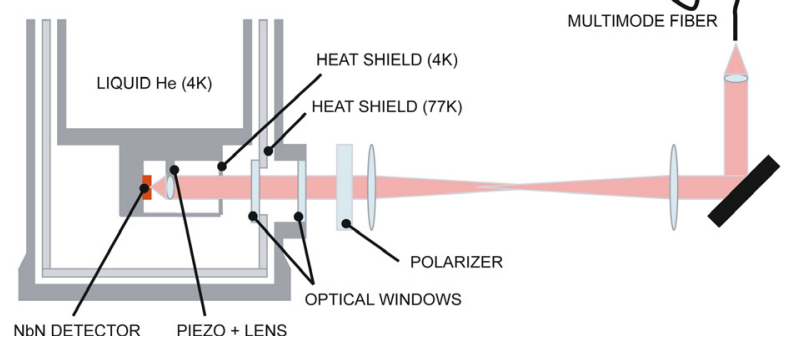

Fig. 1. (Color online) (a) SEM image of a NbN SSPD similar to the one used in the experiments. The $100 \mathrm{~nm}$ wide $\mathrm{NbN}$ line is folded into a meander with an area of $10 \times 10 \mu \mathrm{m}^{2}$. (b) Schematic diagram of the readout electronics. The bias current is provided by a voltage source and a resistor of $400 \mathrm{k} \Omega$. The dashed box contains a phenomenologically equivalent circuit of the detector. (c) Schematic overview of the optical setup. Wavelength-filtered light from a lamp is sent through an optical fiber with a $50 \mu \mathrm{m}$ core, and is imaged onto the detector through a telescope and a movable lens mounted inside the cryostat.

of the resonant enhancement. In fact, for a high index Si or GaAs substrate this factor becomes dominant and an absorption efficiencies of $\sim 70 \%$ can be reached without the need for an optical cavity.

\section{Experimental setup}

In our experiments, we used a commercial NbN SSPD [2], with an area of $10 \times 10 \mu \mathrm{m}^{2}$. The detector consists of a $\sim 4 \mathrm{~nm}$ thick $\mathrm{NbN}$ meander on a $R$-plane sapphire substrate. It has a nominal line width of $100 \mathrm{~nm}$ and a filling factor of $\sim 55 \%$. Figure 1a shows a scanning electron microscope (SEM) image of a detector similar to the one used in the measurements.

We mounted the SSPD in a ${ }^{4} \mathrm{He}-$ cryostat and cooled it to a temperature of $\sim 5 \mathrm{~K}$. The temperature remained constant within $10 \mathrm{mK}$ during each measurement run. Figure $1 \mathrm{~b}$ shows a schematic overview of the electronic circuit used to operate the detector. The detector was biased at $90 \%$ of the critical current through a bias- $T$ with a $400 \mathrm{k} \Omega$ resistor. The equivalent circuit of the detector (dashed box) contains a switch that is closed in the superconducting state. When a photon is absorbed, the switch opens temporarily [5]. The resulting voltage pulse across the detector is amplified $(66 \mathrm{~dB})$ and detected by pulse counting electronics.

Unpolarized light from an incandescent tungsten lamp was wavelength-filtered and sent through a $50 \mu \mathrm{m}$ core size multimode optical fiber. The output of the fiber was

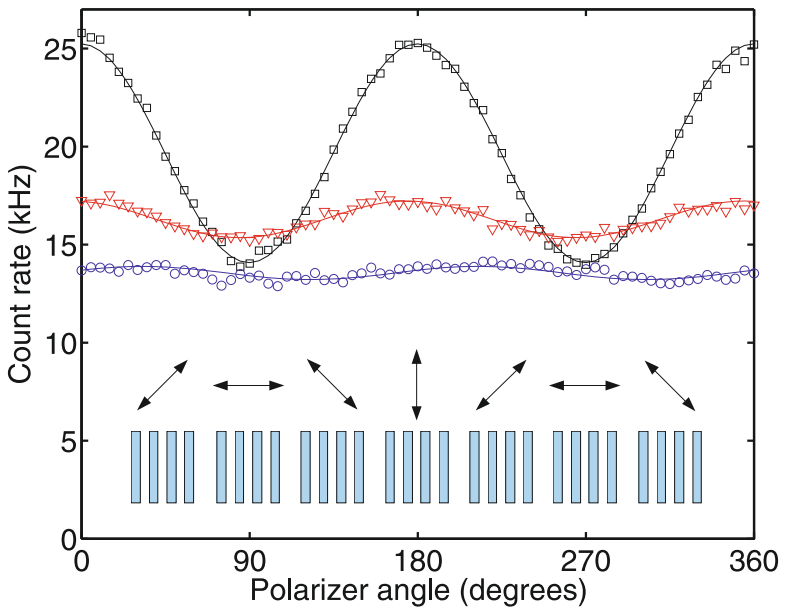

Fig. 2. (Color online) Count rate of the SSPD (corrected for stray light counts) as a function of linear polarization, for a wavelength of $1550 \mathrm{~nm}$ (black squares) and $532 \mathrm{~nm}$ (red triangles). The blue circles show the count rates for $1550 \mathrm{~nm}$ light when two wedge depolarizers (under a relative angle of $45^{\circ}$ ) are placed after the polarizer. The insets show the orientation of the $E$-field relative to the detector, for the different polarizer settings. The solid curves are sinusoidal fits to the data, used to extract the polarization contrast.

imaged onto the detector using a telescope and a lens mounted on a piezo stage inside the cryostat, as shown in Figure 1c. To probe the polarization dependence of the detection efficiency, a linear polarizer with an extinction ratio better than 100:1 for the wavelength range of interest was placed in the parallel part of the beam. To probe the wavelength dependence, we used different narrow bandpass filters ( $\leq 10 \mathrm{~nm}$ FWHM) in combination with several edge filters, to ensure that the light on the detector was monochromatic.

\section{Polarization dependence}

Figure 2 shows the count rate of the detector as a function of linear polarization for a wavelength of $1550 \mathrm{~nm}$ (black squares) and $532 \mathrm{~nm}$ (red triangles). Note that the absolute count rates at different wavelengths cannot be compared directly, due to a difference in incident power. The insets show the orientation of the $E$-field relative to the detector. The measured count rates follow a sinusoidal dependence as a function of polarization and are minimal when the $E$-field is perpendicular to the lines of the detector.

We define the polarization contrast $C$ as

$$
C=\frac{N_{\|}-N_{\perp}}{N_{\|}+N_{\perp}},
$$

where $N_{\|}$and $N_{\perp}$ are the count rates of the detector when the light is polarized parallel and perpendicular to the wires, respectively. This definition of the contrast is a direct measure for the polarization effects, independent of 
the electronic quantum efficiency $\left(\eta_{\mathrm{e}}\right)$, and the incident power. We extract the contrast from the sinusoidal fits to the data (solid curves in Fig. 2). It varies with the wavelength of the incident light and is independent of the bias current and temperature of the detector in our experiment.

The blue circles in Figure 2 show the count rate as a function of polarizer angle, at a wavelength of $1550 \mathrm{~nm}$, when two wedge depolarizers under a relative angle of $45^{\circ}$ were placed after the polarizer. These wedge depolarizers effectively depolarize the incident light by imposing a position-dependent rotation of the polarization. Indeed, the polarization contrast in this case is reduced to below $3 \%$. The lower average count rate can be attributed to the extra four air-glass interfaces in the optical setup, leading to an increased reflection of the incident light.

The polarization effect can be understood by comparing the periodic structure of the detector to that of a wire grid polarizer [13] that consists of a grid of parallel, highly conductive metal wires with a subwavelength spacing. For a perfect conductor the $E$-field should be perpendicular to the metal surface. As a consequence, only light with a polarization perpendicular to the wires is efficiently transmitted. A similar argument holds for lossy metals, albeit that in this case the field penetrates into the metal, leading to absorption. This absorption is largest when the $E$-field is parallel to the wires, since in this case the field penetrates more into the metal.

For the typical dimensions and spacing of the NbN wires, an effective medium approach that is accurate for both polarizations is difficult $[14,15]$. Instead, we calculated the absorption at normal incidence for an infinitelysized detector, using the rigorous coupled-wave analysis (RCWA) developed in reference [16]. This method finds an exact solution of Maxwell's equations by expressing the electromagnetic fields in the different materials as a summation over all diffraction orders. The Fourier components of the periodic dielectric constant couple the diffraction orders in the patterned region. The continuity of the parallel component of the wavevector, together with the boundary conditions for the $E$ and $H$ fields fully determine the field in all regions. From this the intensity in all reflected and transmitted diffraction orders can be calculated. The absorption in the grating is then simply given by $A=1-R-T$, where $R$ and $T$ are the reflected and transmitted intensity.

The effects of focusing of the incident beam can be taken into account by decomposing the beam into plane waves with wave vector $\boldsymbol{k}$. Each of these plane waves will experience a different absorption $A(\boldsymbol{k})$. The effect of finite detector size can be incorporated in a similar way, by multiplying the beam profile in the near field by an aperture function $D(\boldsymbol{r})$ which is 1 at the location of the detector, and 0 elsewhere. Taking both into account, the total absorption is given by the convolution integral

$$
A=\int_{\boldsymbol{k}} A(\boldsymbol{k})[u(\boldsymbol{k}) \cdot D(\boldsymbol{k})]^{2} d \boldsymbol{k}
$$

where $u(\boldsymbol{k})$ is the Fourier transform of the beam profile, and $D(\boldsymbol{k})$ is the Fourier transform of the aperture function $D(\boldsymbol{r})$.

The $k$-spread of the incident waves is determined by the detector size [determining the spread in $D(\boldsymbol{k})$ ] and the numerical aperture of the last lens in the illuminating system, determining the spread in $u(\boldsymbol{k})$. The latter is the most important factor in our experiment, since we used a large- $N A$ lens to focus the incoming light onto the detector. Calculations of the absorption of the grating as a function of angle of incidence (i.e., as a function of $\boldsymbol{k}$ ) show however, that the absorption only varies appreciably from the absorption at normal incidence for angles of incidence corresponding to $N A>0.5$. Therefore, the total absorption given by equation (2) can be approximated by a product of the absorption coefficient at normal incidence and the total intensity impinging on the (finite-sized) detector. This justifies the use of a plane wave calculation in the rest of this paper.

To calculate the absorption efficiency, we used the nominal structure parameters of the detector, and tabulated values of the dielectric constant of the sapphire substrate $\left(n_{\text {sapphire }}=1.74\right.$ at $\left.1550 \mathrm{~nm}\right)$ [17]. For the wavelength-dependent dielectric constant of $\mathrm{NbN}$, a Drude model [18] was used, giving a refractive index $n_{\mathrm{NbN}}=$ $5.5+6.3 i$ at a wavelength of $1550 \mathrm{~nm}$. This value is close to the value reported in reference [7], for a thicker $\mathrm{NbN}$ film.

Figure 3a shows the calculated absorption for polarization parallel (blue line) and perpendicular (red line) to the wires, as a function of wavelength. The absorption for parallel-polarized light monotonously increases with wavelength, whereas the absorption for perpendicular polarization goes through a maximum and decreases for wavelengths above $800 \mathrm{~nm}$. This leads to a higher polarization contrast for longer wavelengths.

For comparison, the dashed line in Figure 3a shows the absorption of an unpatterned film, multiplied by the filling factor of $\mathrm{NbN}$, as was suggested in reference [1]. This estimate deviates over the entire wavelength range from the polarization-averaged result obtained by RCWA, which shows that for structures with features smaller than the wavelength of light, a more refined model is needed. We will discuss this refined model in Section 4. The fact that the absorption decreases for both the parallel polarization and for the closed film is mostly due to dispersion of the dielectric constant of the $\mathrm{NbN}$ material, $\epsilon_{\mathrm{NbN}}$.

In Figure $3 \mathrm{~b}$ we compare the measured polarization contrast (red dots) to the results of the calculations (black solid curve), as a function of wavelength. For comparison, the calculated contrast is shown for filling factors of $52 \%$ (dashed curve) and 58\% (dash-dotted curve) as well. The experimentally observed contrast varies between $\sim 5 \%$ and $\sim 30 \%$ and increases with wavelength. The error bars on the experimental points represent slight variations in the measured polarization contrast during different measurement runs, as well as a slight polarization in the illuminating light source, of $\sim 1 \%$. We attribute the fact that the calculation and the measurements differ for lower 

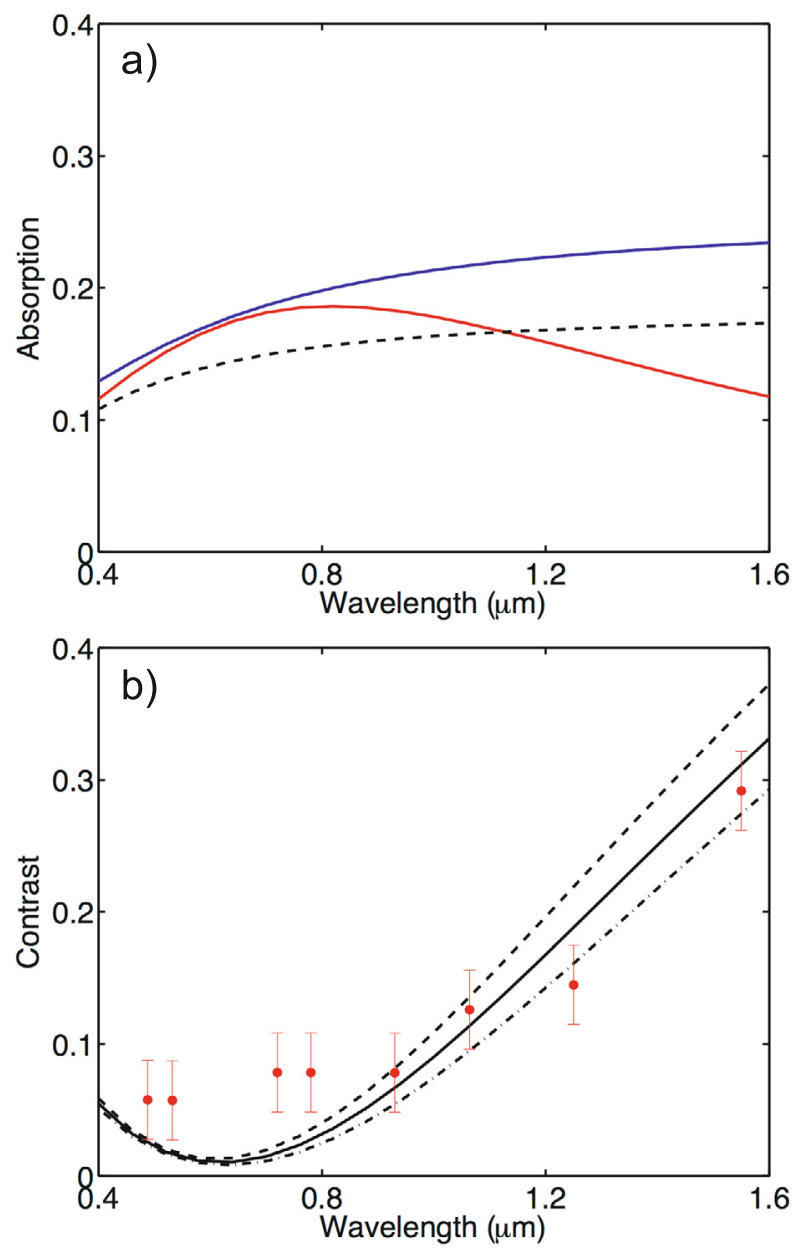

Fig. 3. (Color online) (a) Calculated absorption efficiency of a NbN grating as function of wavelength for polarization parallel (blue curve) and perpendicular (red curve) to the lines of the detector. For comparison, the dashed line shows the calculated absorption of an unpatterned film multiplied by the filling factor of NbN. (b) Measured (red dots) and calculated (black curves) polarization contrast as a function of wavelength. The calculations are shown for a filling factor of $52 \%$ (dashed), $55 \%$ (solid), and $58 \%$ (dash-dotted) and a film thickness of $4.5 \mathrm{~nm}$.

wavelengths to the fact that we used literature values for the dielectric constant of NbN. It is known that the dielectric constant of $\mathrm{NbN}$ varies as a function of the deposition parameters [18] and may depend on the film thickness as well [19]. Additional calculations (not shown) reveal, that for lower wavelengths, the polarization contrast is increasingly sensitive to small variations in the dielectric constant of $\mathrm{NbN}$.

It has been shown that the linear-polarization dependence can be removed by changing the design of the detector [11]. A spiraling detector breaks the translational symmetry that causes the strong polarization contrast. The optical absorption in such a detector, however, will be lower than the maximum obtainable for parallel-polarized light, due to the fact that in these detectors, partial screening of the electric field is always possible.

\section{An optical impedance model}

In order to gain some physical insight into the absorption in the detector, we start out by describing the absorption of a film of thickness $d$ with a complex dielectric constant $\epsilon_{2}$, embedded between two dielectrics with refractive index $n_{1}$ and $n_{3}$, respectively. The film is illuminated from the medium with index $n_{1}$.

We can define the optical impedance of a medium $i$ with refractive index $n_{i}$ as

$$
\eta_{i}=\frac{\eta_{0}}{n_{i}}
$$

where $\eta_{0}=\sqrt{\mu_{0} / \epsilon_{0}}=377 \Omega$ is the impedance of the vacuum. The reflection and transmission of the layered system are given by [20]

$$
\begin{aligned}
& R=\left|\frac{\eta_{\text {load }}-\eta_{1}}{\eta_{\text {load }}+\eta_{1}}\right|^{2}, \\
& T=\frac{\eta_{1}}{\eta_{3}}\left|\frac{2 \eta_{\text {load }}}{\eta_{\text {load }}+\eta_{1}}\right|^{2},
\end{aligned}
$$

where $\eta_{\text {load }}$ is the combined load impedance of the film and the backing substrate. The absorption of the film is again given by $A=1-R-T$.

If we assume that the film is thin enough to neglect interference effects $\left(k_{0} d \ll 1\right)$, the load impedance is given by $[9]$

$$
\eta_{\text {load }} \approx \frac{R_{\square} \eta_{3}}{R_{\square}+\eta_{3}},
$$

where $R_{\square} \approx \eta_{0} / k_{0} d \operatorname{Im} \epsilon_{2}$ is the square resistance for a highly absorbing $\left(\operatorname{Im} \epsilon_{2} \gg \operatorname{Re} \epsilon_{2}\right)$ film, and $k_{0}$ is the wave vector of the light in vacuo. With these assumptions, we can write the absorption in the film as

$$
\begin{aligned}
A & =\frac{4}{\eta_{1} R_{\square}}\left(\frac{\eta_{1} R_{\square} \eta_{3}}{\eta_{1}+R_{\square}+\eta_{3}}\right)^{2} \\
& =4 n_{1} \frac{k_{0} d \operatorname{Im} \epsilon_{2}}{\left(n_{1}+n_{3}+k_{0} d \operatorname{Im} \epsilon_{2}\right)^{2}} .
\end{aligned}
$$

The absorption of the film reaches a maximum value $A_{\max }=n_{1} /\left(n_{1}+n_{3}\right)$ for a square resistance given by

$$
R_{\square}=\frac{\eta_{1} \eta_{3}}{\eta_{1}+\eta_{3}}
$$

Note that the maximum possible absorption is a function of the refractive indices of the surrounding media only. The optimal value of $R_{\square}$ to reach this maximum can be obtained by tuning the film thickness $d$.

\subsection{The effect of film thickness}

Figure 4 shows the absorption and the polarization contrast of a film of $\mathrm{NbN}$, embedded between air $\left(n_{1}=1\right)$ and sapphire $\left(n_{3}=1.74\right)$, as a function of the film thickness. The solid curves show the calculated absorption using the 
E.F.C. Driessen et al.: Optical impedance model for NbN superconducting single-photon detectors

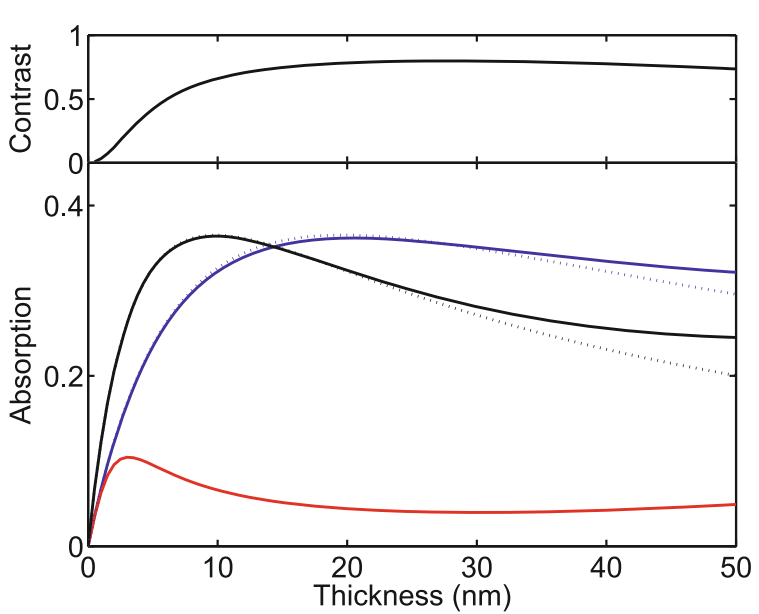

Fig. 4. (Color online) Calculated absorption at a wavelength of $1550 \mathrm{~nm}$ as a function of film thickness. The black curve gives the absorption for a closed film, the blue and red curves for a detector with lattice period $200 \mathrm{~nm}$ and filling factor 0.5 , for polarization parallel (blue) and perpendicular (red) to the wires of the detector. The dotted curves are calculated using the impedance model of Section 4, whereas the solid curves are exact calculations using RCWA. The top graph shows the calculated polarization contrast.

rigorous coupled-wave analysis described before, while the dotted curves are obtained from the impedance model.

For a closed film (black curves), there is a distinct maximum of absorption, that occurs at a thickness

$$
d=\frac{n_{1}+n_{3}}{k_{0} \operatorname{Im} \epsilon_{2}} .
$$

For thinner films, the transmission through the film is too high to get maximal absorption, whereas for thicker films, reflection dominates.

The blue and red curves in Figure 4 show the absorption for a detector with filling factor 0.5 and lattice period $200 \mathrm{~nm}$, for polarization parallel and perpendicular to the wires, respectively. The thickness for which the absorption in the patterned film is maximum, is higher than the optimal thickness for the closed film. The dotted line is calculated using the impedance model of Section 4, taking an effective dielectric constant for the absorbing film, given by [14]

$$
\epsilon_{\mathrm{eff}}=(1-f) \epsilon_{\mathrm{slits}}+f \epsilon_{\mathrm{NbN}}
$$

where $f$ is the filling factor of the metal, and $\epsilon_{\text {slits }}$ is the dielectric constant of the material in the slits, typically air. Since only the imaginary part of $\epsilon_{\mathrm{eff}}$ determines the absorption in the film, the absorption of the detector can simply be calculated by multiplying the thickness of the film by the filling factor. For the polarization perpendicular to the wires of the detector, it is not so straightforward to define an effective dielectric constant for the patterned film $[14,15]$. For this polarization the light is concentrated in the air slits and the effective dielectric constant is closer to that of air. Therefore the condition $\operatorname{Im} \epsilon_{2} \gg \operatorname{Re} \epsilon_{2}$ used

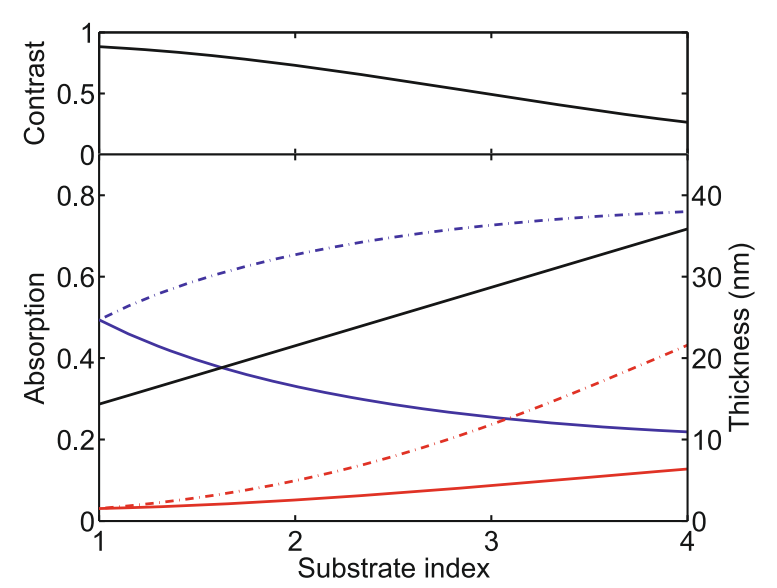

Fig. 5. (Color online) Calculated absorption for a detector with filling factor 0.5 , lattice period $200 \mathrm{~nm}$, at a wavelength of $1550 \mathrm{~nm}$, as a function of the substrate refractive index. The solid curves are for illumination from the air side, the dash-dotted curves for illumination from the substrate side. The blue and red curves give the absorption for polarization parallel and perpendicular to the wires, respectively. The detector thickness is changed at each substrate index, to achieve maximal absorption. The thickness is given by the black line (right axis). The top graph shows the polarization contrast.

to define the impedance model, breaks down for this polarization.

Surprisingly, the calculation also shows that it is easily possible to construct a detector where the absorption for parallel polarization is larger than the absorption of an unpatterned film of the same thickness. Since the electronic efficiency of the detector, $\eta_{\mathrm{e}}$, strongly depends on the thickness of the metal $[21,22]$, it is important to realize that the absorption for parallel-polarized light is a function of $d f \operatorname{Im} \epsilon_{2}$. A reduction in thickness of the detector, to increase the electronic efficiency, can thus be countered by increasing the filling factor accordingly.

\subsection{Illuminating through sub- or superstrate}

Commonly, NbN SSPDs are deposited on a substrate of sapphire and illuminated from air. An inspection of equation (7) shows that for a certain choice of sub- and superstrate, a factor of $n_{3} / n_{1}$ in absorption can be gained by illuminating the detector from the medium with the higher refractive index. Figure 5 shows the calculated absorption for a detector, with a superstrate of air $\left(n_{1}=1\right)$, as a function of the refractive index of the substrate. The thickness of the detector is set such that maximal absorption in the detector is achieved. This thickness is indicated with the black line. The solid curves give the absorption for illumination from the air, whereas the dash-dotted curves give the absorption for illumination from the substrate. The blue and red curves are for polarization parallel and perpendicular to the wires, respectively. We stress that this effect is caused by a lower impedance mismatch and 
should be separated from the cavity enhancement of the absorption, previously reported in references $[10,12]$.

When the substrate index is increased, the absorption rises for illumination from the substrate side. For illumination from the air side, the absorption for parallel polarization decreases. Note however that in both cases, the polarization contrast decreases, from $C=0.88$ at $n_{3}=1$ to $C=0.26$ at $n_{3}=4$, and is independent on the direction of illumination, as shown in the top graph of Figure 5 . The absorption is a factor of $n_{3}$ higher, when the detector is illuminated from the substrate, as expected from the impedance model. It is interesting to note that, for parallel-polarized light, the absorptions from super- and substrate add up to give $A_{\text {super }}+A_{\text {sub }} \approx 1$. It is therefore possible to construct a detector with higher absorption, up to $70 \%$, and lower polarization contrast, by using a high refractive index substrate (e.g. Si or GaAs) and illuminating the detector from the substrate. Unfortunately, increasing the refractive index of the substrate also increases the wavelength for which diffraction orders in the substrate appear. The first diffraction order at normal incidence appears at $\lambda / a=n_{3}$, with $a$ the periodicity of the structure, and $\lambda$ the wavelength of the light. In general, these diffraction orders lower the absorption efficiency. For a typical lattice period of $200 \mathrm{~nm}$, and a substrate index of $n_{3}=3.5$, the first diffraction order appears at a wavelength of $700 \mathrm{~nm}$, making detectors on a high-refractiveindex substrate less effective for detecting visible light. The problem of diffraction could be circumvented by designing a detector that has a variable line spacing.

\section{Conclusion}

We have measured a strong polarization dependence in the detection efficiency of NbN superconducting single photon detectors and find a wavelength dependent polarization contrast between $5 \%$ and $30 \%$. This effect can be explained by the geometry of the detector. Calculations of the optical absorption efficiency give good agreement with the measured data. We have demonstrated that the polarization dependence can be removed by the use of wedge depolarizers.

Furthermore, we have shown that the parameters of the detector can be tuned to achieve an absorption for a polarization parallel to the detector wires, that exceeds the absorption of an unpatterned film of the same thickness. We have given a simple optical impedance model, that allows for a quick estimate of the parameters needed to optimize the detector. For parallel-polarized light, the maximum absorption achievable is not determined by the thickness or the dielectric constant of the metal film, nor by the filling factor, but only by the refractive indices of the surrounding media. We have shown that by illuminating the detector from the substrate it is possible to increase the detection efficiency of the detector even further, by a factor equal to the refractive index of the substrate. Such highly absorbing, highly polarization-dependent detectors can be employed to efficiently detect photons with a well-defined polarization.
We thank Jos Disselhorst, Jennifer Kitaygorsky, Teun Klapwijk, Hans Mooij, and Raymond Schouten for technical assistance and discussions. This research was financed by the Dutch Foundation for Scientific Research (NWO) and the Foundation for Fundamental Research of Matter (FOM).

\section{References}

1. G.N. Gol'tsman, O. Okunev, G. Chulkova, A. Lipatov, A. Semenov, K. Smirnov, B.M. Voronov, A. Dzardanov, C. Williams, R.R. Sobolewski, Appl. Phys. Lett. 79, 705 (2001)

2. G.N. Gol'tsman, O. Minaeva, A. Korneev, M. Tarkhov, I. Rubtsova, A. Divochiy, I. Milostnaya, G. Chulkova, N. Kaurova, B.M. Voronov et al., IEEE Trans. Appl. Supercond. 17, 246 (2007)

3. H. Takesue, S.W. Nam, Q. Zhang, R.H. Hadfield, T. Honjo, K. Tamaki, Y. Yamamoto, Nat. Photon. 1, 343 (2007)

4. A. Semenov, A. Engel, K. Il'in, G.N. Gol'tsman, M. Siegel, H.-W. Hübers, Eur. Phys. J. Appl. Phys. 21, 171 (2003)

5. A.J. Kerman, E.A. Dauler, W.E. Keicher, J.K.W. Yang, K.K. Berggren, G.N. Gol'tsman, B.M. Voronov, Appl. Phys. Lett. 88, 111 (2006)

6. M. Ejrnaes, R. Cristiano, O. Quaranta, S. Pagano, A. Gaggero, F. Mattioli, R. Leoni, B. Voronov, G.N. Gol'tsman, Appl. Phys. Lett. 91, 262509 (2007)

7. A. Engel, A. Semenov, H.-W. Hübers, K. Il'in, M. Siegel, Physica C 444, 12 (2006)

8. M. Bell, N. Kaurova, A. Divochiy, G.N. Gol'tsman, J. Bird, A. Sergeev, A.A. Verevkin, IEEE Trans. Appl. Supercond. 17, 267 (2007)

9. K.E. Kornelsen, M. Dressel, J.E. Eldridge, M.J. Brett, K.L. Westra, Phys. Rev. B 44, 11882 (1991)

10. V. Anant, A.J. Kerman, E.A. Dauler, J.K.W. Yang, K.M. Rosfjord, K.K. Berggren, Opt. Expr. 16, 10750 (2008)

11. S.N. Dorenbos, E. Reiger, N. Akopian, U. Perinetti, V. Zwiller, T. Zijlstra, T.M. Klapwijk, Appl. Phys. Lett. 93, 161102 (2008)

12. K.M. Rosfjord, J.K.W. Yang, E.A. Dauler, A.J. Kerman, V. Anant, B.M. Voronov, G.N. Gol'tsman, K.K. Berggren, Opt. Expr. 14, 527 (2006)

13. G.R. Bird, M. Parrish, J. Opt. Soc. Am. 50, 886 (1960)

14. D.E. Aspnes, Am. J. Phys. 50, 704 (1982)

15. J.M. Pitarke, F.J. Garcia-Vidal, Phys. Rev. B 57, 15261 (1998)

16. M.G. Moharam, E.B. Grann, D.A. Pommet, T.K. Gaylord, J. Opt. Soc. Am. A 12, 1068 (1995)

17. E.D. Palik, Handbook of Optical Constants of Solids, Vol. III (Academic Press, 1998)

18. K. Tanabe, H. Asano, Y. Katoh, O. Michikami, J. Appl. Phys. 63, 1733 (1988)

19. W.-J. Lee, J.-E. Kim, H.Y. Park, S. Park, M.-S. Kim, J.T. Kim, J.J. Ju, J. Appl. Phys. 103, 073713 (2008)

20. S. Ramo, J.R. Whinnery, Fields and Waves in Modern Radio, 2nd edn. (John Wiley \& Sons, 1953)

21. A. Verevkin, A. Pearlman, W. Slysz, J. Zhang, M. Currie, A. Korneev, G. Chulkova, O. Okunev, P. Kouminov, K. Smirnov et al., J. Mod. Opt. 51, 1447 (2004)

22. A. Jukna, J. Kitaygorsky, D. Pan, A.S. Cross, A.J. Pearlman, I. Komissarov, O. Okunev, K. Smirnov, A. Korneev, G. Chulkova et al., Acta Phys. Pol. A 113, 955 (2008) 